\title{
Functionalization of Multi-Walled Carbon Nanotube and Mechanical Property of Epoxy-Based Nanocomposite
}

\author{
Wesley Francisco ${ }^{1}$, Filipe Vargas Ferreira1, Eduardo Vargas Ferreira², Luciana de Simone Cividanes ${ }^{1}$, \\ Aparecido dos Reis Coutinho ${ }^{3}$, Gilmar Patrocínio Thim ${ }^{1}$
}

\begin{abstract}
The focus of this study is to evaluate the effect of carboxyl and amino functionalization of multiwalled carbon nanotubes on the mechanical property of the epoxy resin filled with modified carbon nanotubes. The carbon nanotubes were treated with sulfuric and nitric acids and also with hexamethylenediamine. The presence of acid and amine chemical groups on the carbon nanotube surface was confirmed by X-ray photoelectron spectroscopy. The composites were produced with epoxy resin and modified carbon nanotubes. Vickers hardness tests were carried out on the composites and neat resin. The results showed an increase of hardness in the composite prepared with functionalized carbon nanotubes. This phenomenon is due to the fact that the chemical interaction between modified carbon nanotube and epoxy resin is much stronger than between pristine carbon nanotube and epoxy resin. This stronger interaction is related to the presence of functionalized carbon nanotubes.
\end{abstract}

KEYWORDS: Epoxy matrix composite, Carbon nanotubes, Vickers hardness.

\section{INTRODUCTION}

Carbon nanotubes (CNTs) were discovered in 1991 by Sumio Iijima (Iijima, 1991) and they have attracted a worldwide attention due to their outstanding thermal, electronic and mechanical properties (Shin et al. 2013; Wang and Liew 2015). Therefore, one of the most promising applications is as reinforcing fillers (Shen et al. 2007; Bhuiyan et al. 2013).

The introduction of CNTs in the polymer matrix started in 1994. Since then, investigations have been addressed to improving the polymer properties when compared to the base polymer (Saeb et al. 2015). The effects of CNTs on rheological, thermal, electrical and mechanical properties of thermoset polymers are well known, but in the recent years epoxy resin have brought more attention due to its extremely good chemical and corrosion resistance, acceptable adhesion behavior and remarkable mechanical characteristics (Li and Shimizu 2009; Chen et al. 2014).

The composite filled with CNTs have attracted the attention of aeronautics industries, since it offers a great weight reduction if a metallic material is replaced by nanocomposites. The superior strength-to-weight ratio provided by this material results in the reduction of fuel consumption, which is one of the major challenges for the aerospace industry, such as military and commercial aircrafts, space vehicles, satellites, and others (Gohardani et al. 2014).

However, the CNT application as polymer filler has been hindered by some difficulties in the dispersion and low

1.Departamento de Ciência e Tecnologia Aeroespacial - Instituto Tecnológico de Aeronáutica - Divisão de Ciências Fundamentais - São José dos Campos/SP - Brazil. 2.Universidade Federal de Juiz de Fora - Departamento de Estatística - Juiz de Fora/MG - Brazil. 3.Universidade Metodista de Piracicaba - Laboratório de Materiais Carbonosos - Piracicaba/SP - Brazil.

Author for correspondence: Wesley Francisco | Instituto Tecnológico de Aeronáutica | Praça Marechal Eduardo Gomes, 50 - Vila das Acácias | CEP: $12.228-900$ São José dos Campos/SP - Brazil | Email: wesleyf@ita.br

Received: 04/08/2015 | Accepted: 06/24/2015 
adhesion to matrix (Disfani and Jafani 2013). These facts limit significantly the CNT applications, since CNT tend to agglomerate due to Van der Waals force (Ratna et al. 2013). Researchers have added functional groups on the CNT surface to improve its dispersion. Many different kinds of CNT functionalization have been reported, such as carboxyl and amino groups, and these functionalizations improved the CNT dispersion in polymers (Wang et al. 2007; Qiu and Wang 2010).

The focus of this paper is to evaluate the effect of carboxyl and amino functionalization of CNTs on the mechanical property of the epoxy resin filled with modified CNTs. The functionalized CNTs were characterized by $\mathrm{X}$-ray photoelectron spectroscopy (XPS). Moreover, functionalized CNTs were added to the polymer matrix and the Vickers hardness tests were carried out on the neat resin, composite with pristine CNT and composites with modified CNTs.

\section{EXPERIMENTAL MATERIALS}

The CNTs used in this study were acquired from Laboratório Associado de Sensores e Materiais - Instituto Nacional de Pesquisas Espaciais (LAS/INPE) and prepared by a chemical vapor deposition method, as described in a previously study (Antunes et al. 2011). CNT was purified, performed an oxidative acid treatment and then the functionalization step was performed (Jin et al. 2011). The functionalized sample was labeled as CNT-H.

\section{SURFACE MODIFICATION AND FUNCTIONALIZATION OF CARBON NANOTUBES}

A quantity of CNT-H (0.8 g) was added to $90 \mathrm{~mL}$ of sulfuric acid $\left(\mathrm{H}_{2} \mathrm{SO}_{4}\right.$, Merck, 98\%) and $30 \mathrm{~mL}$ (v/v 3:1) of nitric acid $\left(\mathrm{HNO}_{3}\right.$, Vetec, $\left.70 \%\right)$, in an ultrasonicator bath with power of $225 \mathrm{~J} / \mathrm{s}$ for $6 \mathrm{~h}$ at room temperature. This specimen was called CNT-Ac.

The specimen CNT-Ac (0.3 g) was dispersed in $150 \mathrm{~mL}$ of hexamethylenediamine ( $\mathrm{HMDA}, \mathrm{NH}_{2}\left(\mathrm{CH}_{2}\right)_{6} \mathrm{NH}_{2}$, Aldrich, $70 \%)$. The mixture was maintained under stirring and heating at $100{ }^{\circ} \mathrm{C}$ for 4 days. Then, the modified CNTs were filtered through $0.45 \mu \mathrm{m}$ of polytetrafluorethilene (PTFE) and they were named as CNTHMDA. Figure 1 presents an illustration of surface modification.

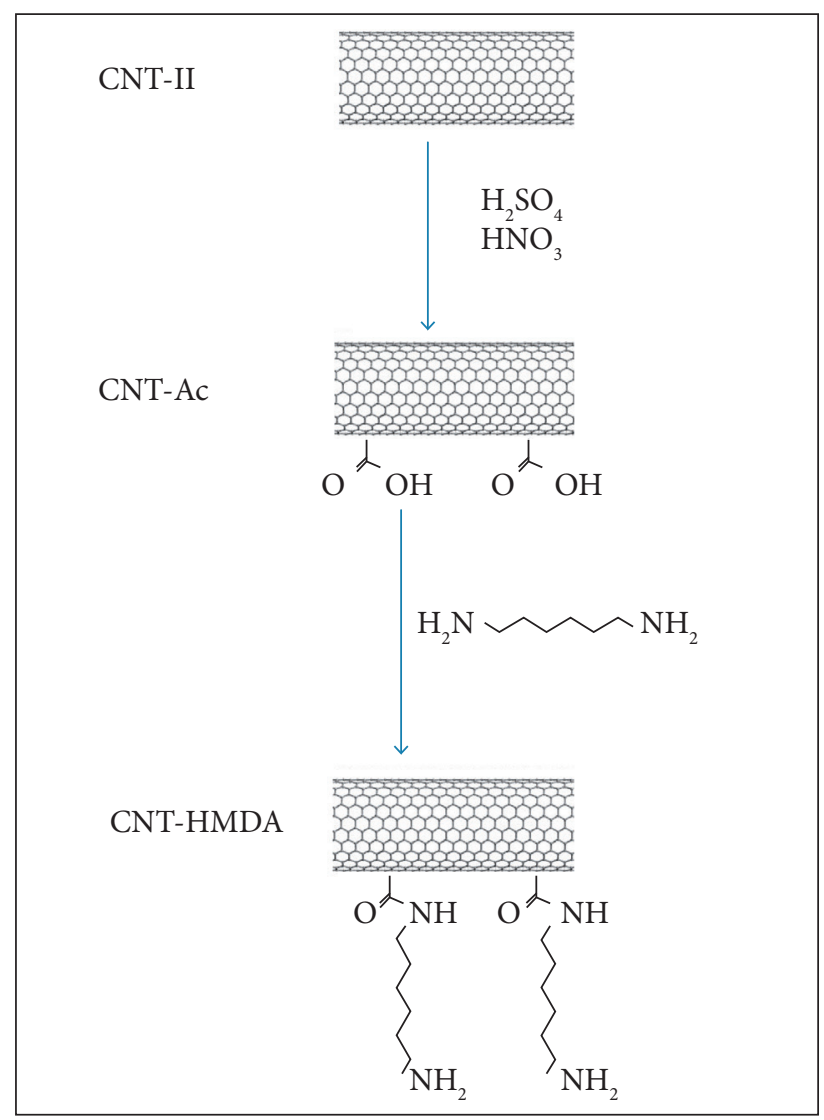

Figure 1. Surface modification and functionalization.

\section{PREPARATION OF NANOCOMPOSITES AND NEAT RESIN}

The nanocomposites (CNT-H/epoxy, CNT-Ac/epoxy and CNT-HMDA/epoxy) and the neat resin (epoxy) were synthesized with epoxy resin diglycidyl ether of bisphenol A (DGEBA), diaminodiphenylmethane (DDM) and $0.2 \%$ wt of CNT.

\section{CHARACTERIZATION AND MEASUREMENTS}

XPS analysis of the CNT samples was carried out on a commercial spectrometer (UNI-SPECS UHV), with Mg Ka line $(h v=1253.6 \mathrm{eV})$ and a pass energy set at $10 \mathrm{eV}$. Vickers hardness measurements were carried out on samples in a diamond Vickers indenter Tester FM-700, where a $0.2 \mathrm{kgf}$ load was applied for $10 \mathrm{~s}$. The hardness value HV (in GPa) was calculated from the indentation load and the diagonal of the Vickers imprint. Thirty two indents were made on each surface keeping an appropriate distance from the sample edges and between indentation marks, avoiding boundary effects (confidence intervals: 95\%). The entire computational environment was developed in R program, a statistical computational and graphical environment. 


\section{RESULTS AND DISCUSSION}

Figure 2 shows the XPS analysis on the samples CNT-H, CNT-Ac and CNT-HMDA. The deconvolution of XPS C $1 \mathrm{~s}$ spectra shows a main component at $284.4 \mathrm{eV}$, which is related to aromatic carbon bonding $\left(\mathrm{C}-\mathrm{C} \mathrm{sp}{ }^{2}\right)$ from the hexagon walls of the CNTs. The peak centered at $285.2 \mathrm{eV}$ refers to $\mathrm{C}-\mathrm{CH} \mathrm{sp}^{3}$ bond, which is related to defects in the aromatic structure and that ranging $285.2-285.9 \mathrm{eV}$ may be related to hydrocarbon from surface contamination. In addition, other peaks may be assigned to chemical bindings of $\mathrm{C}-\mathrm{O}$ at $286.3 \mathrm{eV}, \mathrm{C}=\mathrm{O}$ at $287.7 \mathrm{eV}$ and $\mathrm{O}-\mathrm{C}=\mathrm{O}$ at $289 \mathrm{eV}$. The plasmon $\pi-\pi^{*}$ peak has placed at $291 \mathrm{eV}$. The deconvolution of XPS N 1s spectra (Fig. 2d) shows three peaks: $\mathrm{C}-\mathrm{NH}_{2}$ at $399.1 \mathrm{eV} ; \mathrm{N}-\mathrm{C}$ at $400.3 \mathrm{eV}$ and N-O at $402 \mathrm{eV}$.

The evidence on the XPS spectra in Figs. $2 \mathrm{a}$ and $2 \mathrm{~b}$ is that the sample CNT-Ac has a higher percentage of $\mathrm{C}-\mathrm{O}, \mathrm{C}=\mathrm{O}$ and $\mathrm{O}-\mathrm{C}=\mathrm{O}$ groups than the sample $\mathrm{CNT}-\mathrm{H}$, which indicates the presence of carboxyl group on the CNT-Ac surface. In addition, Fig. $2 c$ shows the presence of $\mathrm{C}-\mathrm{N}$ chemical group, indicating the presence of nitrogenated functional group on the CNT surface. The deconvolution of XPS C1s spectra (Figs. 2a, 2b and $2 \mathrm{c}$ ) shows that the amount of chemical groups related to peak at $285.2 \mathrm{eV}\left(\mathrm{C}-\mathrm{CH} \mathrm{sp}{ }^{3}\right)$ increases in the following order:
$\mathrm{CNT}-\mathrm{H}<\mathrm{CNT}-\mathrm{Ac}<\mathrm{CNT}-\mathrm{HMDA}$. This increase is related to an increase in the defects on the CNTs wall, which contributes for adding functional groups on the CNT-Ac and CNT-HMDA surfaces (Figs. 2b and 2c) (Komarova et al. 2015).

The distribution of hardness results can be seen in Fig. 3. It is noticed that the hardness measurements are randomly distributed in the confidence intervals, where the data normality (p-value < 0.001) was admitted.

Figure 4 shows the box-plot of different composites $(\mathrm{CNT}=0.2 \mathrm{wt} . \%)$. It is noticed from Figs. $4 \mathrm{a}$ and $4 \mathrm{~b}$ that the adding of CNT in the resin without surface modification produces a slight increase in the average hardness from 21.33 to $21.69 \mathrm{HV}$ (significantly same values; $p$-value $=0.487$ ). It is known that functionalized CNTs have more disorganized microstructure compared to pristine CNT, which leads to greater dispersion of CNTs, hence functionalized CNTs have better interfacial bonding with the polymer matrix (Cividanes et al. 2012). In addition, Figs. 4c and 4d show an increase in the average hardness of about $30 \%$ in the composite prepared with CNT-Ac. Afterwards, in the CNT-HMDA sample, the hardness increased from 27.95 to 29.56 (statistically different values; $\mathrm{p}$-value $=0.06$ ). It may be attributed to the presence of amine functional groups onto CNT surfaces (Liu and Wagner 2005; Martinez-Hernandez et al. 2010; Gkikas and Paipetis 2015).

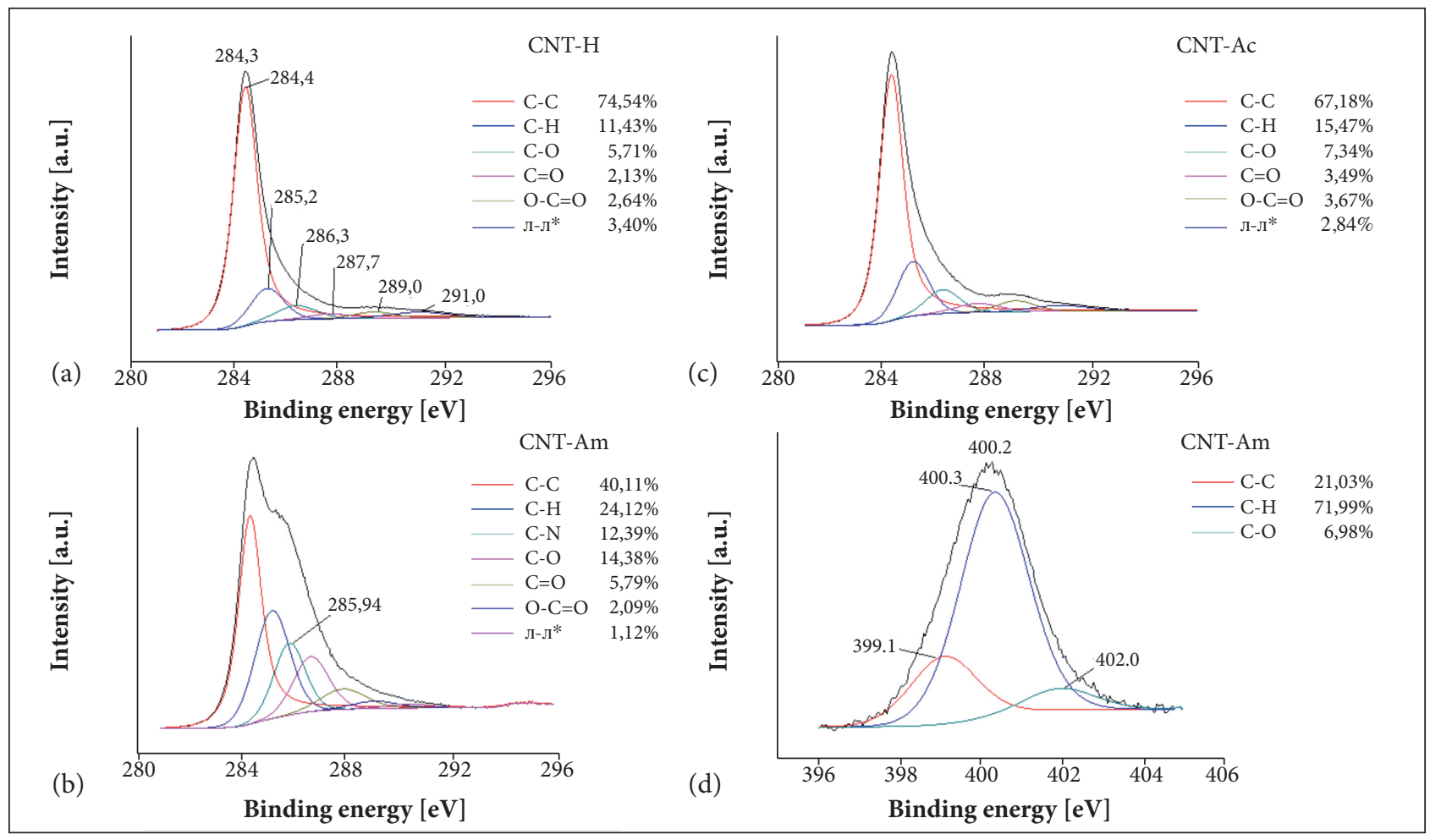

Figure 2. XPS spectra regarding (a) CNT- H C 1s; (b) CNT- Ac C 1s; (c) CNT- HMDA C 1s; (d) CNT- HMDA N1s. 


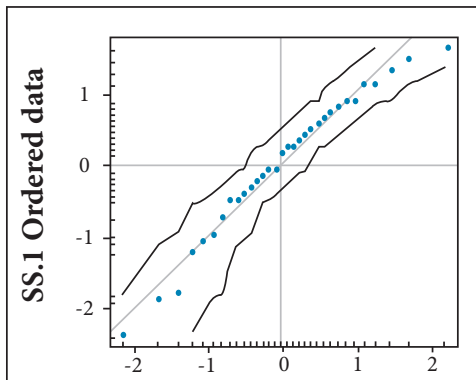

(a)

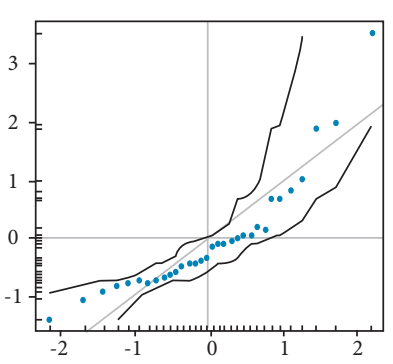

(b)

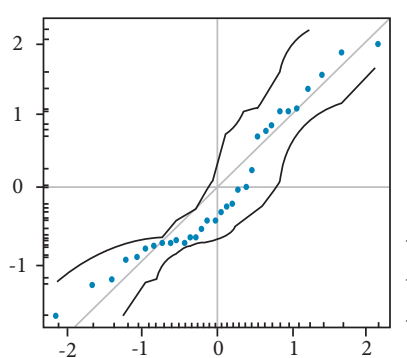

(c)

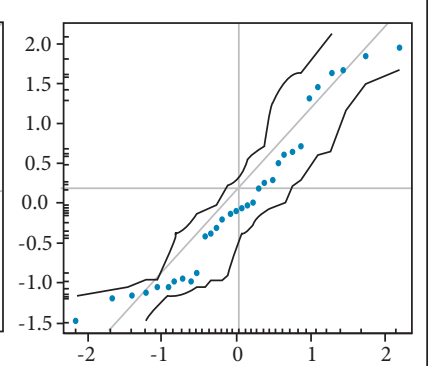

(d)

Normal quantities

Figure 3. Normal QQ Plot. (a) Epoxy; (b) CNT- H/epoxy; (c) CNT- Ac/epoxy; (d) CNT- HMDA/epoxy.

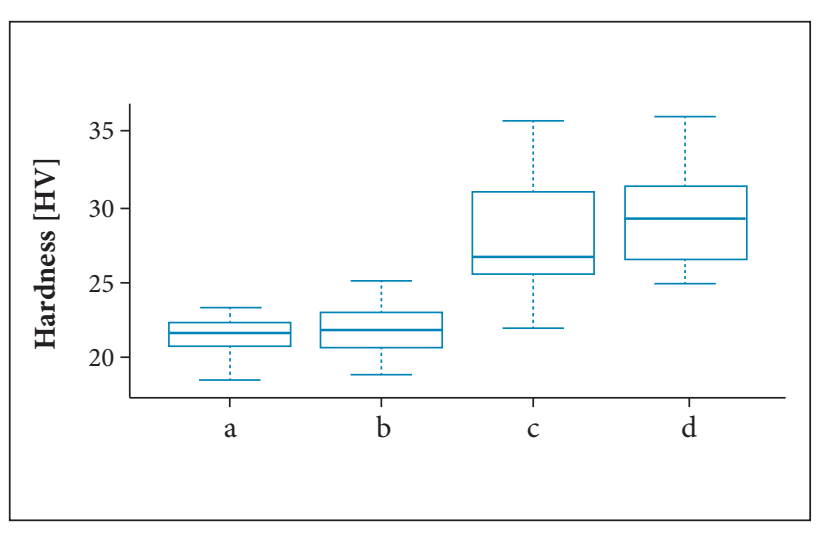

Figure 4. Vickers hardness of the samples: (a) Epoxy; (b) CNT- H/epoxy; (c) CNT- Ac/epoxy; (d) CNT- HMDA/epoxy.

On the other hand, it appears that CNT additions significantly increase the composite sample variability in the hardness ( $p$-value $<0.001$ ). The high variability of the hardness results may be attributed to CNTs dispersion in the polymer matrix, which can affect the final nanocomposite properties (Song and Young 2005; Bal and Samal 2006; Cividanes et al. 2012).

The average hardness of the CNT-HMDA/epoxy composite is higher than the average hardness of the CNT-Ac/epoxy composite. However, if we analyze the maximum deviation values, both hardness seem to be closer.

\section{CONCLUSIONS}

The CNTs functionalized with acid and HMDA were submitted to XPS analysis and it was observed the presence of the carboxyl and amine groups, which confirms the functionalization success. The results showed that the neat resin and CNT-H composite have almost the same hardness. The functionalization has increased the hardness of CNT-Ac and CNT-HMDA composites, regardless of the maximum deviation values of CNT-Ac and CNT-HMDA composites are almost the same, although the amine-functionalized composite has presented the highest average hardness value among all composites.

\section{ACKNOWLEDGEMENTS}

The authors acknowledge Conselho Nacional de Desenvolvimento Científico e Tecnológico (CNPq - Grant 141197/2014-5) and Fundação de Amparo à Pesquisa do Estado de São Paulo (FAPESP — Grant 2013/20218-0, 2013/23042-0) for financial support, as well as Laboratório Associado de Sensores e Materiais - Instituto Nacional de Pesquisas Espaciais and Laboratório de Espectroscopia de Fotoelétrons - Universidade Estadual Paulista, for collaboration.

\section{REFERENCES}

Antunes E, Resende V, Mengui U, Cunha J, Corat E, Massi M (2011) Analyses of residual iron in carbon nanotubes produced by camphor/ferrocene pyrolysis and purified by high temperature annealing. Appl Surf Sci 257(18):8038-8043. doi: 10.1016/ j.apsusc.2011.04.090

Bal S, Samal SS (2006) Influence of dispersion states of carbon nanotubes on mechanical properties of epoxy nanocomposites.
Proceedings of the Recent Trends in Mechatronics, Nanotechnology and Robotics; Rourkela, India.

Bhuiyan A, Pucha RV, Worthy J, Karevan M, Kalaitzidou K (2013) Defining the lower and upper limit of the effective modulus of CNT/polypropylene composites through integration of modeling and experiments. Compos Struct 95:80-87. doi: 10.1016/ j.compstruct.2012.06.025 
Chen Y, Wang S, Pan F, Zhang J (2014) A numerical study on electrical percolation of polymer-matrix composites with hybrid fillers of carbon nanotubes and carbon black. J Nano Mat 2014(2014):article ID 614797. doi: 10.1155/2014/614797

Cividanes L, Brunelli D, Antunes E, Corat E, Sakane K, Thim G (2012) Cure study of epoxy resin reinforced with multiwalled carbon nanotubes by raman and luminescence spectroscopy. J Appl Polym Sci 127(1):544-553. doi: 10.1002/app.37815

Disfani MN, Jafani SH (2013) Assessment of intertube interactions in diferent functionalized multiwalled carbon nanotubes incorporated in a phenoxy resin. Polym Eng Sci 53:168-175. doi: 10.1002/ pen. 23244

Gkikas G, Paipetis AS (2015) Optimisation and analysis of the reinforcement effect of carbon nanotubes in a typical matrix system. Meccanica 50:461-478. doi 10.1007/s11012-014-9915-z

Gohardani O, Elola MC, Elizetxea C (2014) Potential and prospective implementation of carbon nanotubes on next generation aircraft and space vehicles: a review of current and expected applications in aerospace sciences. Prog Aerosp Sci 70:42-68. doi: 10.1016/ j. paerosci.2014.05.002

lijima $S$ (1991) Helical microtubules of graphitic carbon. Nature 354:56-58. doi: 10.1038/354056aO

Jin FL, Rhee KY, Park SJ (2011) Functionalization of multi-walled carbon nanotubes by epoxide ring-opening polymerization. J Solid State Chem 184(12):3253-3256. doi: 10.1016/j.jssc.2011.10.021

Komarova NS, Krivenko AG, Ryabenko AG, Naumkin AV, Maslakov KI, Savilov SV (2015) Functionalization and defunctionalization of single walled carbon nanotubes: electrochemical and morphologic consequences. J Electroanal Chem 738:27-34. doi: 10.1016/ j.jelechem.2014.11.028

Li Y, Shimizu H (2009) Toward a stretchable, elastic, and electrically conductive nanocomposite: morphology and properties of poly[styrene-b-[ethylene-co-butylene)-b-styrene]/multiwalled carbon nanotube composites fabricated by high-shear processing. Macromolecules 42(7):2587-2593. doi: 10.1021/ma802662c

Liu L, Wagner HD (2005) Rubbery and glassy epoxy resins reinforced with carbon nanotubes. Compos Sci Technol 65(11-12):1861-1868. doi: 10.1016/j.compscitech.2005.04.002

Martinez-Hernandez AL, Velasco-Santos C, Castaño VM (2010) Carbon nanotubes composites: processing, grafting and mechanical and thermal properties. Curr Nanosci 6:12-39. doi: $10.2174 / 157341310790226270$

Qiu J, Wang S (2010) Reaction kinetics of functionalized carbon nanotubes reinforced polymer composites. Mater Chem Phys 121 (1-2):295-301. doi: 10.1016/j.matchemphys.2010.01.039

Ratna D, Jagtap SB, Rathor R, Kushwaha RK, Shimpi N, Mishra SN (2013) A comparative studies on dispersion of multiwall carbon nanotubes in poli (ethylene oxide) matrix using dicarboxylic acid and amino acid based modifiers. Polym Composite 34(6):1003-1011. doi: $10.1002 /$ pc. 22508

Saeb MR, Najafi F, Bakhshandeh E, Khonakdar HA, Mostafaiyan M, Simon F, Scheffler C, Mäder E (2015) Highly curable epoxy/ MWCNTs nanocomposites: an effective approach to functionalization of carbon nanotubes. Chem Eng J 259:117-125. doi: 10.1016/ j.cej.2014.07.116

Shen J, Huang W, Wu L, Hu Y, Te M (2007) The reinforcement role of different amino-functionalized multi-walled carbon nanotubes in epoxy nanocomposites. Compos Sci Technol 67(15-16):3041-3050. doi: 10.1016/j.compscitech.2007.04.025

Shin H, Yang S, Chang S, Yu S, Cho M (2013) Multiscale homogenization modeling for thermal transport properties of polymer nanocomposites with Kapitza thermal resistance. Polymer 54(5):1543-1554. doi: 10.1016/j.polymer.2013.01.020

Song YS, Young JR (2005) Influence of dispersion states of carbon nanotubes on physical properties of epoxy nanocomposites. Carbon 43:1378-1385.

Wang JF, Liew KM (2015) On the study of elastic properties of CNTreinforced composites based on element-free MSL method with nanoscale cylindrical representative volume element. Compos Struct 124:1-9.

Wang S, Liang R, Wang B, Zhang C (2007) Epoxide-terminated carbon nanotubes. Carbon 45:3042-3059. doi: 10.1016/j.carbon. 2007.10.014 